\title{
Mild recessive epidermolytic hyperkeratosis associated with a novel keratin 10 donor splice-site mutation in a family of Norfolk terrier dogs
}

\author{
K.M. Credille, K.F. Barnhart, J.S. Minor and R.W. Dunstan* \\ Comparative Dermatology Laboratory, Texas A\&M University, College Station, TX, U.S.A. \\ *Pfizer Global Research and Development, Ann Arbor, MI, U.S.A.
}

\section{Summary}

\section{Correspondence \\ Kelly Credille, Eli Lilly and Co., 2001 West Main Street GL44, Greenfield, IN 46160, U.S.A. \\ E-mail:credillekm@lilly.com}

\section{Accepted for publication}

9 December 2004

Key words:

animal model, epidermolysis, genodermatosis, keratin, mutation

\section{Conflicts of interest:}

None declared.

This work was performed at Michigan State University in East Lansing, MI, U.S.A. and Texas A\&M University, College Station, TX, U.S.A.
Background Epidermolytic hyperkeratosis in humans is caused by dominant-negative mutations in suprabasal epidermal keratins 1 and 10. However, spontaneous keratin mutations have not been confirmed in a species other than human.

Objectives To describe an autosomal recessive, mild, nonpalmar/plantar epidermolytic ichthyosis segregating in an extended pedigree of Norfolk terrier dogs due to a splice-site mutation in the gene encoding keratin 10 (KRT10).

Methods Dogs were evaluated clinically, and skin samples were examined by light and electron microscopy. Genomic DNA samples and cDNA from skin RNA were sequenced and defined a mutation in KRT10. Consequences of the mutation were evaluated by assessing protein expression with immunohistochemistry and Western blotting and gene expression with real-time RT-PCR (reverse transcriptasepolymerase chain reaction).

Results Adult dogs with the disease had generalized, pigmented hyperkeratosis with epidermal fragility. Light microscopic examination defined epidermolysis with hyperkeratosis; ultrastructural changes included a decrease in tonofilaments and abnormal filament aggregation in upper spinous and granular layer keratinocytes. Affected dogs were homozygous for a single base GT $\rightarrow$ TT change in the consensus donor splice site of intron 5 in KRT10. Keratin 10 protein was not detected with immunoblotting in affected dogs. Heterozygous dogs were normal based on clinical and histological appearance and keratin 10 protein expression. The mutation caused activation of at least three cryptic or alternative splice sites. Use of the cryptic sites resulted in transcripts containing premature termination codons. One transcript could result in shortening of the proximal portion of the 2B domain before the stutter region. Quantitative real-time PCR indicated a significant decrease in KRT10 mRNA levels in affected dogs compared with wild-type dogs.

Conclusions This disease is the first confirmed spontaneous keratin mutation in a nonhuman species and is the first reported recessive form of epidermolytic hyperkeratosis.
Keratin intermediate filaments are essential for structural stability of epidermal keratinocytes and normal stratum corneum formation. Thus, mutations of epidermal keratin genes are largely characterized by haphazard filament aggregation and cell fragility, often resulting in cell lysis (epidermolysis) that is more severe with exposure to trauma. ${ }^{1-4}$ In most mutations of the superficial keratins (KRT1, 10, 2e) blisters and hyperkeratosis are both present. Blistering is most apparent in chil- dren, where the disease is often called bullous congenital ichthyosiform erythroderma, while scaling is more prominent in adults, where the same disease can also be called epidermolytic hyperkeratosis (EHK) ${ }^{5}$

Many different allelic variations occur in genetic disorders of superficial keratins. A recent review listed 22 mutations in KRT1 and 38 in KRT10. ${ }^{6}$ A majority of these mutations are missense mutations and occur in two 'hot spot' regions of the 
molecule: the helix initiation and helix termination motifs. Most keratin mutations are transmitted by an autosomal dominant mode of inheritance. ${ }^{6}$

With the number of keratin mutations that have been identified in humans, and their distinctive clinical and histological features, it is surprising that no spontaneous keratin defect has been confirmed in a species other than human. To date, the recognition of animals with a phenotype of a spontaneous keratinization defect has occurred only in dogs. A Labrador Retriever, ${ }^{7}$ a mixed breed dog, ${ }^{8}$ and arguably, Cavalier King Charles spaniels ${ }^{9}$ have been noted with hyperkeratosis, epidermolysis and/or perinuclear aggregation of tonofilaments and cytoplasmic vacuolation, but none of these cases was confirmed at the molecular level.

In this report, we confirm the first spontaneous heritable keratin defect described in a species other than human. The disease represents a novel mutation in KRT10 transmitted as a recessive trait through an extended pedigree of Norfolk terrier dogs. ${ }^{10}$ Affected dogs display lesions from birth through adulthood with all of the hallmarks of a mild, superficial, nonpalmar/plantar epidermolytic ichthyosis while heterozygotes are clinically and histologically normal.

\section{Materials and methods}

All procedures relating to animal care in this study were approved by the Clinical Research Review Committee, College of Veterinary Medicine, Texas A \& M University, approval number CRRC 02-25.

\section{Animals}

The clinical disease was recognized in seven affected (four female and three male) Norfolk terrier dogs within an extended pedigree and these dogs were compared with normal dogs of the same breed. Normal, heterozygous and affected dogs were available for sampling throughout the course of the study.

\section{Tissue sampling}

A 6-mm punch biopsy and local anaesthesia were used to obtain skin samples for histological and ultrastructural examination, RNA isolation and protein extraction. Both peripheral blood and cheek swabs were used as sources of genomic DNA.

\section{Light and electron microscopy}

For histology, skin biopsy samples were fixed with $10 \%$ neutral buffered formalin, processed routinely, embedded in paraffin, sectioned at 4-5 $\mu \mathrm{m}$ and stained with haematoxylin and eosin. Immunohistochemistry was performed on formalinfixed skin samples using commercially available antibodies that previously had been shown to display the appropriate crossreactivity with canine antigens: $34 \beta \mathrm{B} 4$ (Enzo, Farmingdale,
NY, U.S.A.) demonstrated K1 expression, and DE-K10 (Dako, Carpinteria, CA, U.S.A.) demonstrated K10 expression. ${ }^{7,11}$ For electron microscopy, formalin-fixed tissues were postfixed with $1 \%$ osmium tetroxide in $0.1 \mathrm{~mol} \mathrm{~L}^{-1}$ phosphate buffer and processed routinely. Sections were stained with aqueous uranyl acetate and lead citrate and examined with a Phillips 301 Transmission Electron Microscope. ${ }^{10}$

\section{Sequencing}

Genomic DNA was extracted from blood leucocytes with the Flexigene DNA kit (Qiagen, Valencia, CA, U.S.A.) as per the manufacturer's protocol. To obtain skin cDNA, punch biopsy samples stored at $-80{ }^{\circ} \mathrm{C}$ and/or in RNALater (Ambion, Austin, TX, U.S.A.) were subjected to total RNA extraction using Trizol Reagent (Gibco BRL, Rockville, MD, U.S.A.) as per the manufacturer's protocol and subsequently, reverse transcription of total RNA was performed using random primers provided in the Pro-Star reverse transcriptase-polymerase chain reaction (RT-PCR) kit (Stratagene, La Jolla, CA, U.S.A.). Primers designed to amplify canine KRT10 sequences from skin cDNA or genomic DNA templates were initially derived from conserved nucleotide regions of human (NM_000421 and X_14487) and mouse (XM_283025) K10. As data were collected, canine-specific primers were designed. For amplification, the Failsafe PCR system (Epicentre, Madison, WI, U.S.A.) was employed according to the manufacturer's protocol. The PCR products were resolved by electrophoresis through agarose gels ranging from $1 \cdot 4 \%$ to $2 \cdot 0 \%$. PCR products with bands of appropriate length, as predicted from human KRT10, were purified directly or by gel extraction (Qiagen). Products were sequenced directly using a Perkin Elmer ABI 373, 377XL or 3100 system. Sequence data were aligned using Sequencher 3.1.1 (Gene Codes Corporation, Ann Arbor, MI, U.S.A.). The putative amino acid sequence was determined using the translation software on the ExPASy Molecular Biology Server (http://us.expasy.org; accessed 13 March 2005).

\section{Mutation detection assay}

Genomic DNA was extracted from buccal epithelial cells with the QIAamp Midi kit (Qiagen). A 660-bp region of genomic DNA spanning the mutation was amplified using the Failsafe PCR system (Epicentre) with buffer J, $1 \cdot 0 \mu \mathrm{L}$ of $0 \cdot 2 \mathrm{mmol} \mathrm{L}{ }^{-1}$ forward primer ( $5^{\prime}$-CCTTCCAGGGATTGTTTCTGC-3'), $1 \cdot 0 \mu \mathrm{L}$ of $0.2 \mathrm{mmol} \mathrm{L}^{-1}$ reverse primer (5'-AAGGAACTGACTACAGAAATCAACAG- $3^{\prime}$ ), and $2 \cdot 0 \mu \mathrm{L}$ of DNA. A restriction digest was performed using $15 \mu \mathrm{L}$ of PCR product, $30 \mu \mathrm{L}$ of water, $5 \cdot 0 \mu \mathrm{L}$ of buffer $\mathrm{R}^{+}$and 1.0 unit of MvaI (Fermentas, Hanover, $\mathrm{MD}$, U.S.A.) at $37{ }^{\circ} \mathrm{C}$ for $1 \mathrm{~h} ; 8.0 \mu \mathrm{L}$ of digestion product was electrophoresed on a $2 \%$ agarose gel.

\section{Immunoblotting}

Keratinocytes were harvested from punch biopsy samples by solubilization in Laemmli-type sample buffer and protein was 
quantified using the BCA assay (Pierce, Rockford, IL, U.S.A.). $\beta$-Mercaptoethanol (4\%) was added to extracts after quantification. Protein (12 $\mu \mathrm{g}$ per sample) was electrophoresed on a $10 \%$ Tris- $\mathrm{HCl} /$ glycine/sodium dodecyl sulphate-polyacrylamide gel and proteins were transferred by overnight electroblotting to nitrocellulose membranes. The membranes were incubated with a $1: 50$ dilution of primary antibody (K10, clone RKS60) (Chemicon, Temecula, CA, U.S.A.). After washing in phosphate-buffered saline, the membrane was incubated with a 1:2500 dilution of goat antimouse IgG conjugated with alkaline phosphatase (Sigma, St Louis, MO, U.S.A.). After washing, the phosphatase activity was detected with the chromogenic substrate BCIP/NBT (Sigma).

\section{TaqMan ${ }^{\circledR}$ quantitative real-time polymerase chain reaction analysis}

Total RNA was extracted from 6-mm skin punch biopsy samples from four homozygous normal dogs, four carrier (heterozygous) dogs and four affected dogs with the RNAqueous-micro kit (Ambion). The RNA was subsequently treated with DNasefree (Ambion) to remove genomic DNA contamination, quantified with RiboGreen (Molecular Probes, Eugene, OR, U.S.A.) and stored at $-80^{\circ} \mathrm{C}$. Quantitative real-time RT-PCR was performed with $10 \mathrm{ng}$ of total RNA/reaction and the TaqMan ${ }^{\circledR}$ One-step RT-PCR Master Mix reagents kit (Applied Biosystems, Foster City, CA, U.S.A.). Gene-specific primers for KRT10 (forward primer 5'-CCTGCTTCAGATCGACAATGC-3'; reverse primer $5^{\prime}$-ACCTCGTTCTCATACTTTAATCTGAAGTC-3') and a TaqMan ${ }^{\circledR}$ probe $\left(5^{\prime}\right.$-AGGCTGGCAGCTGA- $\left.3^{\prime}\right)$ containing an MGBNFQ (Minor groove binder/nonfluorescent quencher) 3' label and a $6 \mathrm{FAM}^{\mathrm{TM}} 5^{\prime}$ label (Applied Biosystems) were designed using Perkin-Elmer Applied Biosystems Primer Express software. Primers were designed to cross at least one intron-exon boundary. The highly conserved regions of the keratin genes, particularly the trigger motifs and the $\mathrm{H} 1$ homologous region, were strictly avoided when designing primers to help ensure a high level of specificity. The forward primer was designed to bind to the middle of exon 2. The reverse primer was located at the end of exon 2 and crossed the intron to include the beginning of exon 3. The product size was $66 \mathrm{bp}$. The probe was designed to bind in the region between the primers. A GeneAmp 5700 Sequence Detection System (Applied Biosystems) was used for quantification. The amplification program consisted of an initial $95{ }^{\circ} \mathrm{C}, 10$-min cycle followed by 40 cycles of $95{ }^{\circ} \mathrm{C} 15$-s denaturation and $60{ }^{\circ} \mathrm{C} 1$-min annealing/extension. Samples were analysed in triplicate for the expression of KRT10 and an internal housekeeping gene, 18S ribosomal RNA (Applied Biosystems). The threshold cycle values for each replicate reaction were averaged. All genes were normalized to $18 \mathrm{~S}$ ribosomal RNA, and the fold change was calculated with the formula: $\mathrm{X}=$ $2^{-\Delta \Delta \mathrm{Ct}}$ where $\Delta \Delta \mathrm{Ct}=\left(\mathrm{C}_{\mathrm{t} \text {, target }}-\mathrm{C}_{\mathrm{t} \text {,reference }}\right)_{\text {sample }}-\left(\mathrm{C}_{\mathrm{t} \text {,target }}-\right.$ $\left.\mathrm{C}_{\mathrm{t}, \text { reference }}\right)_{\text {normal }}$. Target refers to the gene of interest, and reference refers to the internal control gene. 'Sample' refers to affected skin, and 'normal' refers to normal skin. ${ }^{12}$

\section{Statistical analysis}

Triplicate measurements of mRNA expression for KRT10 and $18 \mathrm{~S}$ using real-time PCR were obtained from each normal $(n=4)$, carrier $(n=4)$ and affected $(n=4)$ skin biopsy sample. Due to the possibility of interassay variability, a second run was performed on each sample for a total of six measurements. The arithmetic mean of these measurements was obtained and relative differences between KRT10 and 18S calculated. A nonparametric version of the unpaired t-test (Mann-Whitney) was used to compare the relative differences between homozygous normal, heterozygous and affected skin with statistical significance taken at $P<0.05$.

\section{Results}

\section{Clinical description}

An eight-generation pedigree containing 108 dogs was assembled and was consistent with an autosomal recessive mode of inheritance (Fig. 1). The pedigree contained seven affected dogs and 33 obligate and/or genetically confirmed heterozygotes. Clinically, affected dogs were identified within hours after birth by sloughing of the superficial epidermis after mild mechanical trauma to the skin. As adults, the epidermal fragility persisted and was accompanied by hyperpigmentation with generalized dark grey hyperkeratosis; the intertriginous areas were most severely affected (Fig. 2). Erythroderma was not noted. Several affected dogs over 10 years of age were observed and their clinical lesions remained static once they reached adulthood. Footpads, claws, hair and teeth were normal. Heterozygous dogs were clinically normal at all ages.

\section{Light and electron microscopy}

In affected dogs, the most striking histological alteration was the presence of granular cell layer epidermolysis that progressed to areas of intragranular clefting. This was associated with large keratohyaline granules, a minimally to moderately thickened stratum corneum with attenuated corneocytes and mild to moderately papillated epidermal hyperplasia (Fig. 3). None of these changes was present in heterozygotes. Immunohistochemical staining for K10 using formalin-fixed, paraffin-embedded skin biopsy samples failed to define K10 expression in the epidermis of affected dogs, while there was strong, positive staining of the suprabasal epidermis of normal Norfolk terrier dogs, Norfolk terrier dogs heterozygous for the mutation and other normal canine breeds. ${ }^{10}$ Electron microscopy confirmed cytolysis, decreased tonofilaments and abnormal tonofilament clumping in affected dogs.

\section{Mutation detection}

A complete genomic sequence of canine KRT10 (GenBank accession AY318944) was determined using oligonucleotide primers for PCR designed initially from homologous regions 


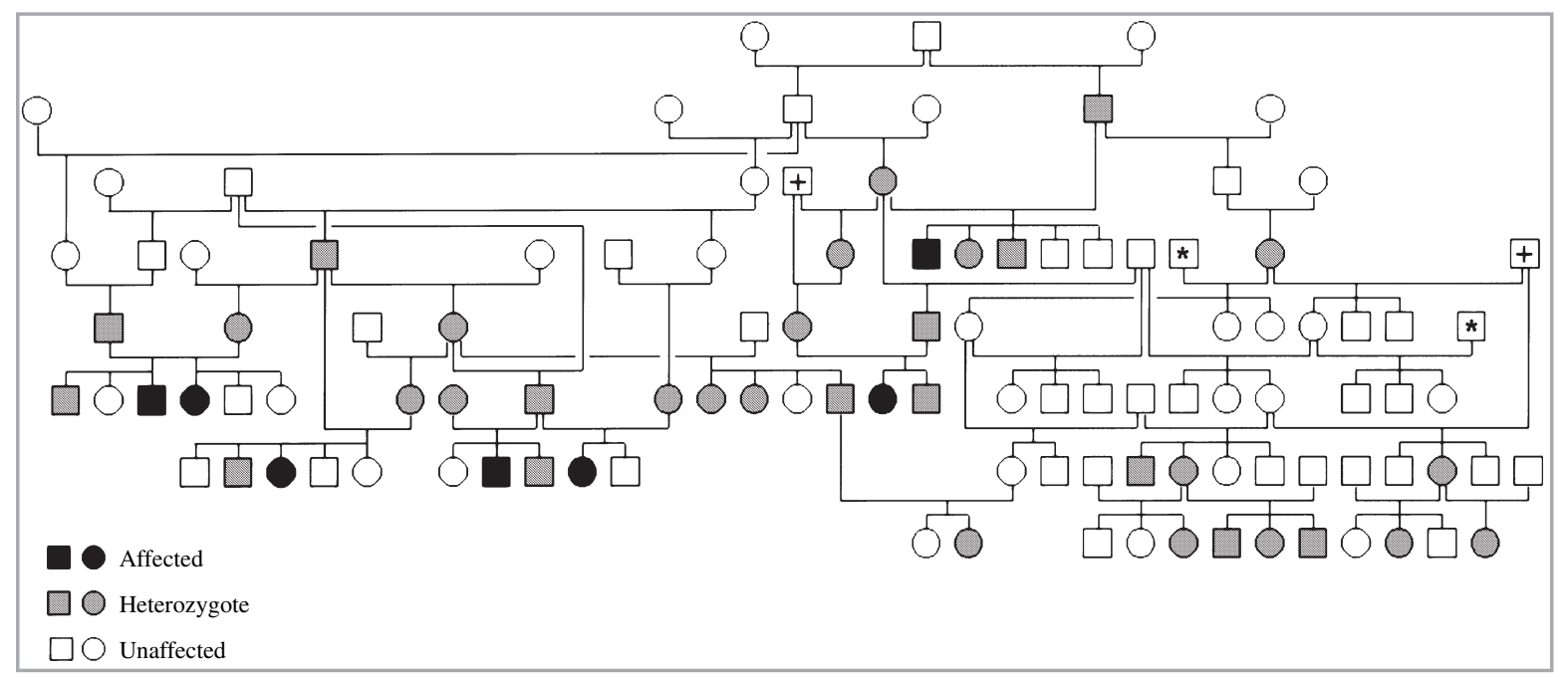

Fig 1. An eight-generation pedigree segregating epidermolytic hyperkeratosis in Norfolk terrier dogs displaying an autosomal recessive mode of inheritance. Note: The symbols + and $*$ indicate the same dog.

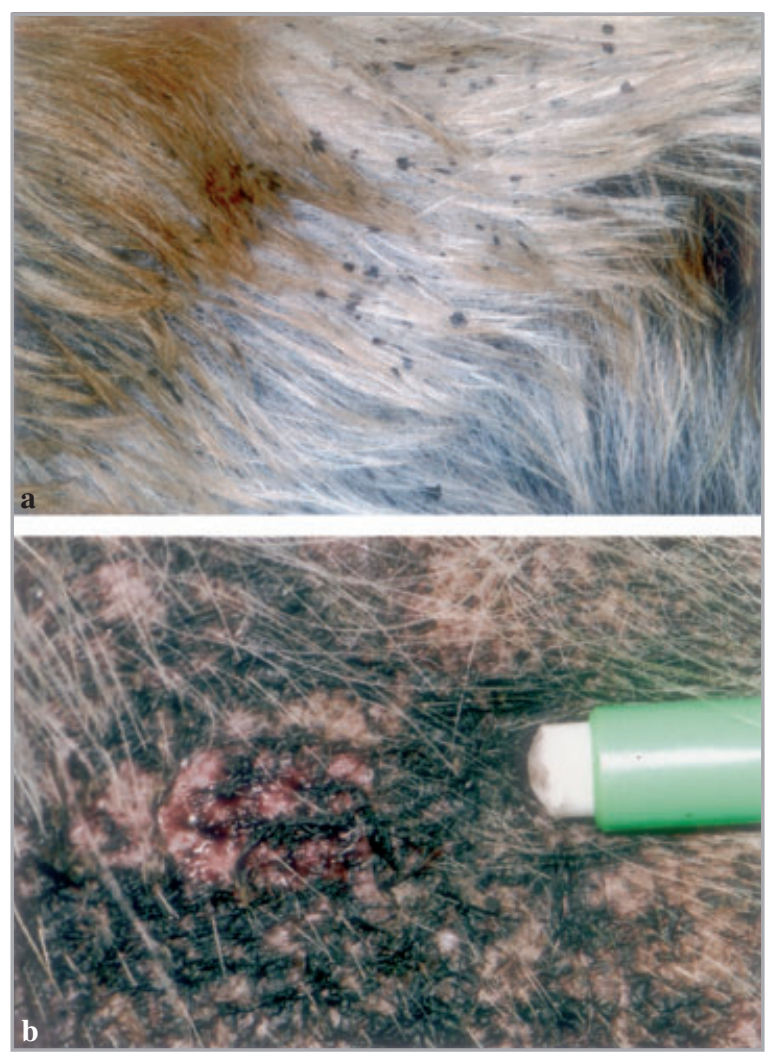

Fig 2. Clinical features of the disease. (a) Moderate black scale is present in the haircoat. (b) The skin is hyperkeratotic and hyperpigmented. Mild mechanical trauma results in superficial bulla formation with subsequent erosion.

of previously reported human and mouse DNA sequences and later from canine sequences. DNA sequencing of a PCR product resulting from amplification of genomic DNA from affected dogs using a canine-specific forward primer in exon 4

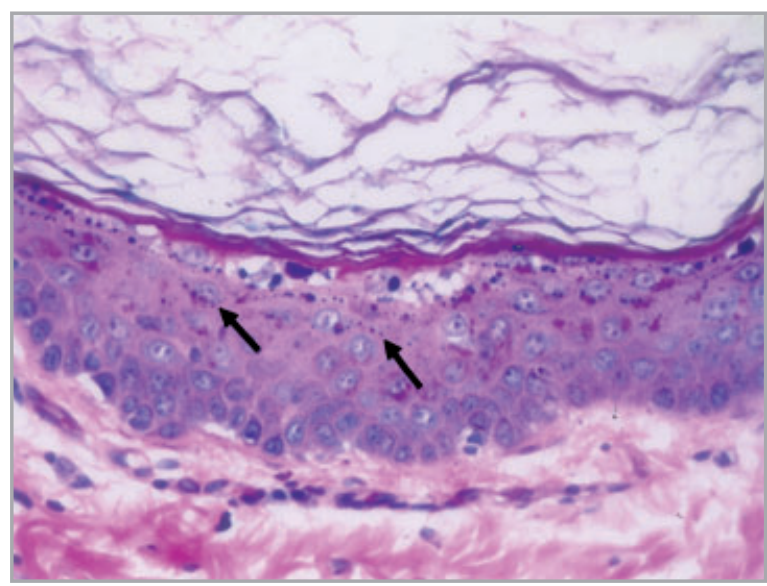

Fig 3. Histological examination of a skin biopsy sample from an affected dog. Characteristic features include epidermal hyperplasia with superficial epidermolysis, large keratohyaline granules, many eosinophilic intracytoplasmic granules, and a hyperkeratotic stratum corneum. Keratohyaline granules are variably present in normal canine epidermis. In contrast to the large keratohyaline granules found in affected dogs, the arrows indicate keratohyaline granules that are more typical in size when these structures are identified. Haematoxylin and eosin, original magnification $\times 400$.

(5'-TCTAGCAGGCTGCGGTAGG-3') paired with a reverse primer in exon 6 (5'-GATGCTGAAGCCTGGTTCAATG-3') revealed a single base $\mathrm{GT} \rightarrow \mathrm{TT}$ change in the consensus donor splice site of intron 5. Sequencing of the same amplification product in DNA samples from parents of affected dogs (obligate heterozygotes) showed that both the wild-type allele and TT mutant allele were present. The expected GT consensus sequence was present in multiple normal Norfolk terrier dogs not from lines related to affected dogs and in multiple other breeds (Fig. 4). No additional nucleotide differences between normal and affected dogs were identified. 


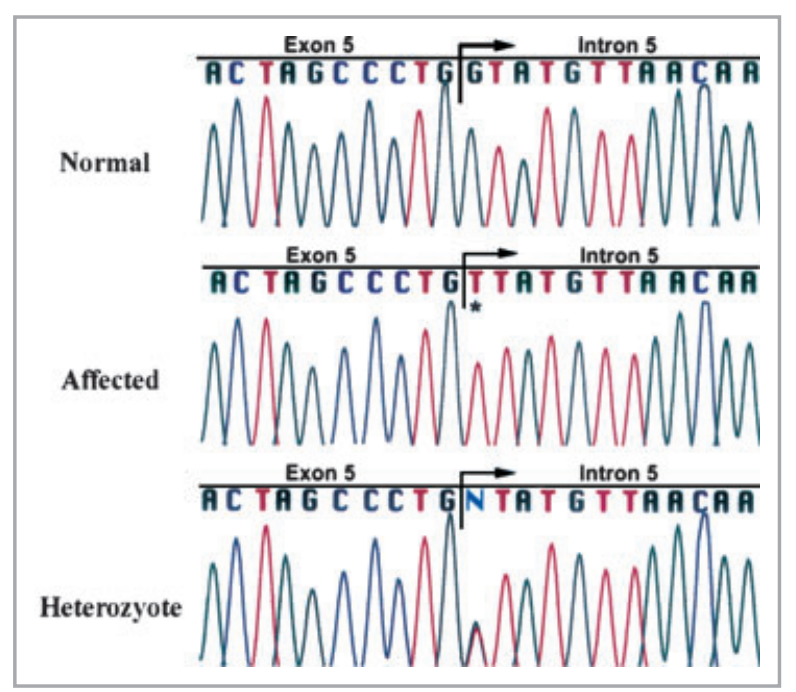

Fig 4. A novel point mutation in the donor splice site of intron 5 (GT $\rightarrow$ TT) causes epidermolytic hyperkeratosis in dogs homozygous for the mutation. Sequencing of KRT10 genomic DNA from normal, affected homozygous and unaffected heterozygous dogs.

To examine the effects of the splice-site mutation, the previously described primer set spanning this site was used to amplify canine KRT10 cDNA from affected and homozygous wild-type Norfolk terrier dogs. Agarose gel electrophoresis revealed an expected single 359-bp band in normal dogs. In affected dogs, the 359-bp band was not detected and multiple smaller and larger bands were identified (Fig. 5). Three of these aberrant amplification products were isolated, re-amplified and sequenced directly. The smallest band (approximately $230 \mathrm{bp}$ ) represented the in-frame and entire removal of exon 5 (126 nucleotides) and could be explained by the alternative use of the normal intron 4 GT site. If translated, this misspliced transcript would produce a deletion of 42 amino acids from the proximal portion of the $2 \mathrm{~B}$ rod domain before the stutter. The brightest amplification product (approximately $330 \mathrm{bp}$ ) corresponded to a 35-bp out-of-frame deletion of the end of exon 5. This probably occurred due to activation of a cryptic splice site within exon 5 (GTCTG). The sequence of this product was out of frame and contained an insertion of 114 incorrect nucleotides followed by a premature termination codon. If translated, the misspliced transcript would encode for 38 incorrect amino acids in the mid 2B domain and then termination of the molecule. Sequencing of a third larger band (approximately $450 \mathrm{bp}$ ) showed it contained a 95-bp out-of-frame inclusion at the $5^{\prime}$ end of intron 5 that was probably due to the use of a GT sequence within intron 5 (GTAAG). This creates a premature termination codon after the insertion of six incorrect nucleotides at the end of exon 5 , truncating the molecule in the mid $2 \mathrm{~B}$ region.

If translated, the abnormal transcripts from these three cryptic/alternative splice sites would result in either a keratin

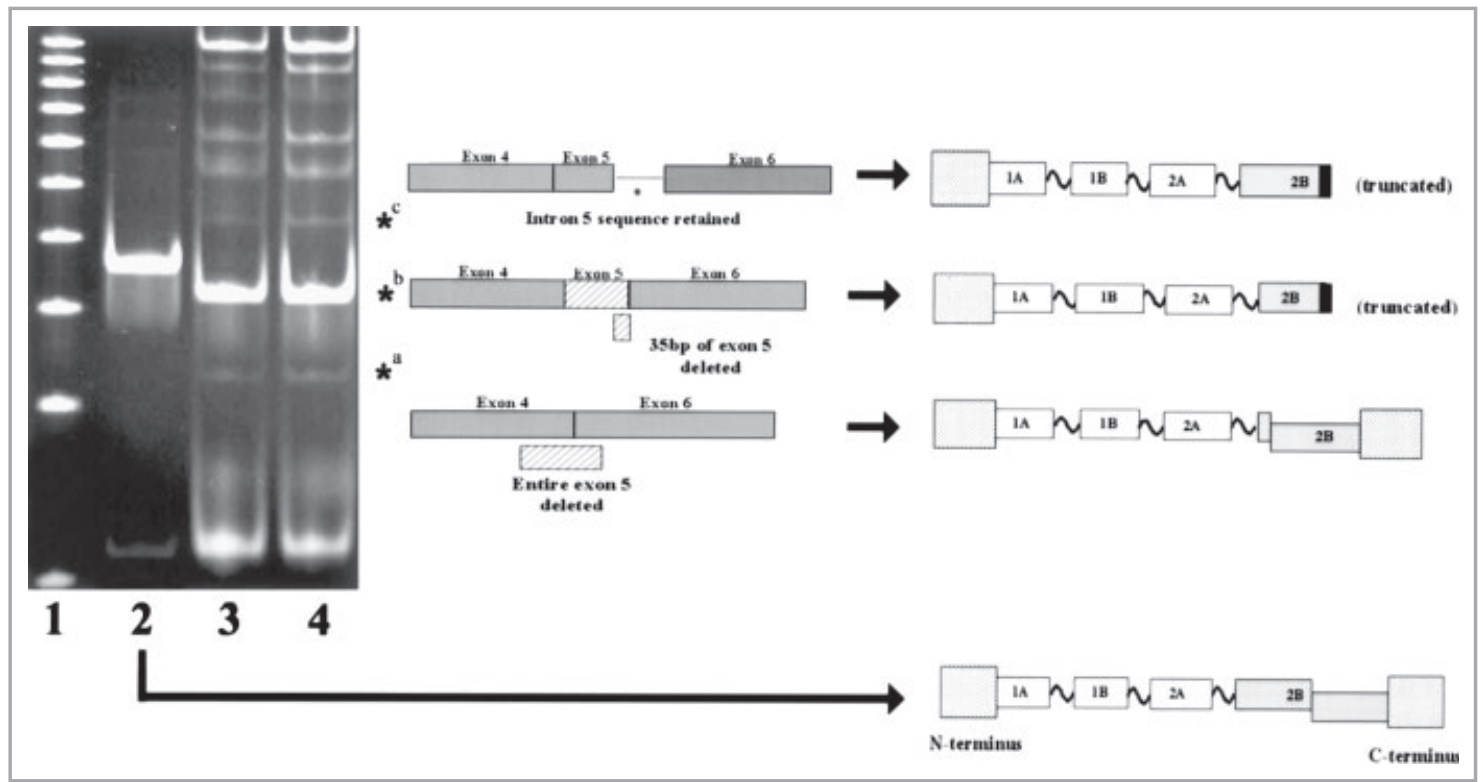

Fig 5. Polymerase chain reaction amplification of KRT10 cDNA in two affected dogs compared with a normal breed-matched dog and corresponding line diagram illustrating the consequences of aberrant splicing of KRT10. Lane 1, 100-bp marker; lane 2, normal dog, expected 359-bp product; lanes 3 and 4 , affected dogs, three aberrant bands $\left(^{*}\right)$ were isolated and sequenced. (*a) The normal donor site in intron 4 is used and as a result exon 5 is removed from the transcript. The nucleotide sequence remains in frame and results in a 42 amino acid deletion from the $2 \mathrm{~B}$ domain. $(* \mathrm{~b})$ A cryptic splice site occurs upstream in exon 5 , resulting in deletion of the last $35 \mathrm{bp}$ of that exon. The resulting sequence is out of frame and 38 incorrect amino acids are encoded before a premature termination codon truncates the molecule in the $2 \mathrm{~B}$ domain. $\left({ }^{*} \mathrm{c}\right)$ An alternative splice site occurs downstream in intron 5, resulting in an additional 95 bp of the intron within the transcript. The sequence is out of frame and generates a termination codon immediately following exon 5, truncating K10 in the 2B domain. 
molecule with a loss of a portion of the $2 \mathrm{~B}$ domain or an incomplete protein that terminates within the mid 2B rod domain (Fig. 5). Other PCR amplification products in Figure 5 in the affected dogs were not examined and may represent other misspliced transcripts or products generated from small amounts of genomic DNA contaminating the cDNA samples.

\section{Screening for the mutation}

The mutation in the intron 5 donor splice site of KRT10 abolishes an MvaI restriction site. Enzyme digestion of a 660-bp PCR product spanning the mutation produces fragments of $247 \mathrm{bp}, 208 \mathrm{bp}$ and $121 \mathrm{bp}$ (and three very small fragments) from the normal allele and fragments of $329 \mathrm{bp}$ and $247 \mathrm{bp}$ in the mutant allele (data not shown). MvaI digests of PCRamplified genomic DNA from the seven affected dogs confirmed that they were homozygous for this mutation. Seven of the obligate heterozygote parents of the affected dogs were available for testing and all were confirmed as heterozygotes. Also, 50 clinically unaffected Norfolk terrier dogs not from the breeding lines in which the disease is segregating and five dogs from other breeds did not possess the nucleotide alteration, indicating it is probably not a polymorphic site in dogs. Using buccal swabs as a source of genomic DNA, this screening test has been made available to breeders of Norfolk terrier dogs.

\section{Keratin 10 protein expression}

From previous work ${ }^{11}$ and based on the predicted amino acid sequence, the molecular weight of canine K10 is $56-58 \mathrm{kDa}$. A band of the predicted size was identified in protein extracts from the skin of homozygous normal Norfolk terriers, in the skin of a confirmed heterozygote and in the normal skin of other breeds (data not shown), but was not found in extracts of the skin of homozygous affected dogs (Fig. 6).

\section{Comparison of KRT10 gene expression}

Quantitative real-time PCR demonstrated a statistically significant decrease $(P<0.05$, Mann-Whitney $)$ in relative mRNA

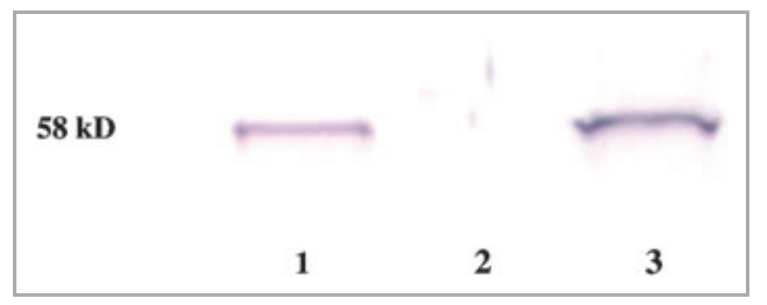

Fig 6. Immunoblot for $\mathrm{K} 10$ in dogs. Lane 1, extract of skin from a homozygous normal Norfolk terrier; lane 2, extract of skin from a homozygous affected Norfolk terrier; lane 3, extract of skin from a heterozygous Norfolk terrier.

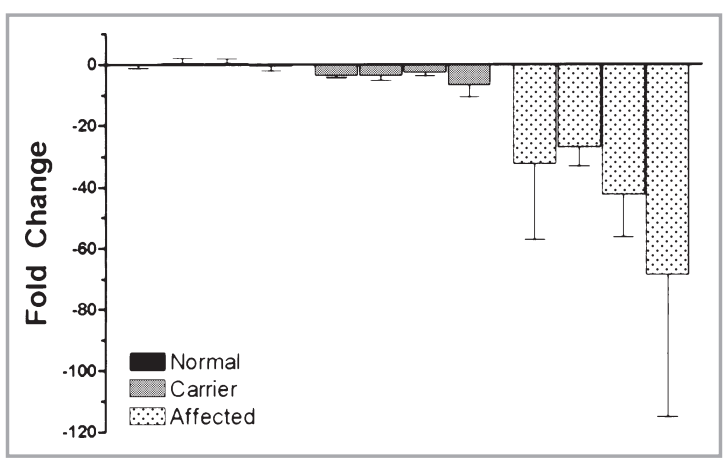

Fig 7. TaqMan ${ }^{\circledR}$ quantitative real-time polymerase chain reaction analysis of KRT10 mRNA comparing four homozygous normal dogs, four heterozygotes and four affected dogs. A significant decrease in KRT10 mRNA was found in the skin of the heterozygous and, to a greater extent, affected dogs.

expression of KRT10 for both affected and carrier dogs compared with homozygous normal dogs (Fig. 7).

\section{Discussion}

In all affected dogs, the phenotype correlated with a homozygous mutation in the GT consensus sequence of the donor splice site in intron 5 of KRT10. This represents the first time a spontaneous keratin mutation has been confirmed in a dog or any mammalian species other than human. Because of the mild phenotype and the prominence of intragranular clefting, we initially considered the disease to have greater phenotypic homology with human ichthyosis bullosa of Siemens; ${ }^{4}$ however, we could not confirm a KRT2e mutation after comparing the canine DNA sequences of normal and affected dogs (data not shown).

The mutation corresponds to the proximal portion of the $2 \mathrm{~B}$ region of the keratin molecule. In humans, a low number of mild EHK phenotypes have been associated with mutations in the 2B domain of KRT1 and KRT10. Mild EHK (KRT10), mild epidermolytic palmar/plantar keratoderma (PPK) (KRT1) and mild combined EHK/PPK (KRT1) have occurred due to mutations in the $2 \mathrm{~B}$ domain upstream from the conserved helix termination motif sequence. ${ }^{13-15}$

As with other structural proteins that form multimers, mutations affecting keratins usually cause a dominant negative effect and recessive forms of these diseases are less commonly recognized. ${ }^{6}$ The majority of recessive keratin disorders cited are forms of epidermolysis bullosa simplex due to mutations in KRT14, ${ }^{6,16,17}$ with a single report in KRT5. ${ }^{18}$ Most of these mutations are nonsense mutations or missense mutations that result in premature termination codons early in the keratin molecule. To our knowledge, the disease in Norfolk terriers is the first confirmed recessive mutation of a suprabasal keratin.

Because of shorter generational times, the importance placed on pedigrees by pure-bred dog breeders, and the obvious clinical features of affected dogs, we were able to confirm the phenotype in an eight-generation pedigree that contained 
seven affected dogs. The pedigree defined an autosomal recessive mode of inheritance. We verified the genotypes of most of the 33 heterozygotes with a mutation detection assay. All heterozygotes were clinically normal. Of the recognized hereditary disorders of the pure-bred dog, approximately $66 \%$ are recessive, ${ }^{19}$ higher than in humans, where approximately $26 \%$ of monogenic diseases are recessive. ${ }^{20}$ This is due to the emphasis on maintaining desirable traits by selective breeding of closely related dogs (inbreeding) and the use of a sire or dam repeatedly when it is viewed as being particularly outstanding. At the same time, an obviously diseased animal would not be bred; therefore, dominant traits are not maintained for long. In sum, the breeding practices associated with pure-bred dogs select for recessive traits but against dominant traits. The recognition of a recessive mutation in a suprabasal keratin, albeit in dogs, indicates that similar recessive mutations may be present in humans but are not recognized due to the rarity of consanguinity in human pedigrees.

The Norfolk terrier KRT10 mutation is associated with the use of multiple exonic and intronic cryptic donor sites and the normal intron 4 donor splice-site sequence as alternative splice sites. In affected dogs, we speculate that lesions result from the combination of markedly decreased filament synthesis, supported by the electron microscopy and immunoblot results, and the production of a small amount of mutated K10 derived from misspliced in-frame transcripts, supported by the presence of abnormal filament aggregates ultrastructurally. Although we could amplify portions of KRT10-derived cDNA from the skin of affected dogs by PCR, the KRT10 mRNA levels were markedly lower than in breed-matched dogs homozygous for the wild-type allele, suggesting that the presence of premature termination codons or other nucleotide alterations leads to nonsense-mediated decay of KRT10 transcripts. If abnormal K10 synthesis does occur, our data indicate at least one mutated form would be altered in the central area of the $2 \mathrm{~B}$ region, not within the helix termination motif.

In this study, examination of confirmed heterozygotes shows that these dogs were normal clinically, histologically and in terms of $\mathrm{K} 10$ protein expression; therefore, it appears that haploinsufficiency did not affect keratin intermediate filament assembly. Additionally, if abnormal K10 protein was produced, it did not disrupt the cytoskeleton sufficiently to produce epidermolysis.

To date, the example most analogous to the Norfolk terrier disease is a genetically modified mouse model with a K10 null mutation. These mice have a milder phenotype than the dogs we describe. ${ }^{21,22}$ The $\mathrm{K} 10^{-/-}$mice were phenotypically normal at birth and, even as adults, did not display epidermolysis, although they did develop epidermal hyperplasia and mild orthokeratosis, presumably related to the effect of the loss of $\mathrm{K} 10$ on cell cycle control. ${ }^{22}$

A second K10 mutant mouse model demonstrates a mild phenotype in the heterozygous state. ${ }^{23}$ This mild phenotype is related to reduced expression of the mutant allele so the wildtype allele can partially compensate for the mutant allele. In the clinically and histologically normal heterozygous dogs we did not define downregulation of the mutant allele, although this may be confirmed in future studies. The murine and canine models differ also in the homozygous state. When homozygous for the 'hot spot' $\mathrm{R} \rightarrow \mathrm{C}$ mutation in exon 1 , the mice have severe epidermolysis resulting in extensive blistering and scaling. ${ }^{23}$

Finally, this represents the first report of a keratin mutation in the intron 5 donor splice site. Splice-site mutations have been reported rarely as causes of EHK and epidermolytic PPK, due to mutations in KRT1, and as causes of epidermolysis bullosa simplex, due to mutations in both KRT5 and 14. The splice-site mutation most similar to this case occurred in the donor site of intron 6 in KRT1 and led to a mild epidermolytic PPK with rare hyperkeratotic lesions in other sites. ${ }^{14}$

As this mutation is outside of the 'hot spot' regions for KRT10, and is recessive, it represents a unique model to further define genotype-phenotype correlations of suprabasal keratin mutations.

\section{Acknowledgments}

This work was supported in part by the Morris Animal Foundation and the Texas A\&M University College of Veterinary Medicine signature programme funds. We would like to thank the Norfolk terrier owners and their veterinarians who have contributed their time and samples for this study.

\section{References}

1 Kirfel J, Magin TM, Reichelt J. Keratins: a structural scaffold with emerging functions. Cell Mol Life Sci 2003; 60:56-71.

2 Ishida-Yamamoto A, McGrath JA, Judge MR et al. Selective involvement of keratins $\mathrm{K} 1$ and $\mathrm{K} 10$ in the cytoskeletal abnormality of epidermolytic hyperkeratosis (bullous congenital ichthyosiform erythroderma). J Invest Dermatol 1992; 99:19-26.

3 Fuchs E. Of mice and men: genetic disorders of the cytoskeleton. Mol Biol Cell 1997; 8:189-203.

4 Irvine $\mathrm{AD}$, McLean WHI. Human keratin diseases: the increasing spectrum of disease and subtlety of the phenotype-genotype correlation. Br J Dermatol 1999; 140:815-28.

5 McLean WHI, Morley SM, Higgins C et al. Novel and recurrent mutations in keratin 10 causing bullous congenital ichthyosiform erythroderma. Exp Dermatol 1999; 8:120-3.

6 Smith FJD. The molecular genetics of keratin disorders. Am J Clin Dermatol 2003; 4:347-64.

7 Mecklenburg L, Hetzel U, Ueberschär S. Epidermolytic ichthyosis in a dog: clinical, histopathological, immunohistochemical and ultrastructural findings. J Comp Pathol 2000; 122:307-11.

8 August JR, Chickering WR, Rikihisa Y. Congenital ichthyosis in a dog: comparison with the human ichthyosiform dermatoses. Compendium Small Anim 1988; 10:40-5.

9 Alhaidari Z, Ortonne J-P, Pisani A. Congenital ichthyosis in two Cavalier King Charles spaniel littermates. Vet Dermatol 1994; 5:11721.

10 Barnhart KF, Credille KM, Ambrus S et al. A heritable keratinization defect of the superficial epidermis in Norfolk terrier dogs. J Comp Pathol 2004; 130:246-54.

11 Walter JH. Cytokeratins in the canine epidermis. Vet Dermatol 2001; 12:81-7. 
12 Lehmann U, Kreipe H. Real-time PCR analysis of DNA and RNA extracted from formalin-fixed and paraffin-embedded biopsies. Methods 2001; 25:409-18.

13 Syder AJ, Yu OC, Paller AS et al. Genetic mutations in the K1 and K10 genes of patients with epidermolytic hyperkeratosis. Correlation between location and disease severity. J Clin Invest 1994; 93: $1533-42$.

14 Hatsell SJ, Eady RAJ, Wennerstrand L et al. Novel splice site mutation in keratin 1 underlies mild epidermolytic palmoplantar keratoderma in three kindreds. J Invest Dermatol 2001; 116:606-9.

15 Terron-Kwiatkowski A, Paller AS, Compton J et al. Two cases of primarily palmoplantar keratoderma associated with novel mutations in keratin 1. J Invest Dermatol 2002; 119:966-71.

16 Ciubotaru D, Bergman R, Baty D et al. Epidermolysis bullosa simplex in Israel: clinical and genetic features. Arch Dermatol 2003; 139:498-505.

17 Lanschuetzer CM, Klausegger A, Pohla-Gubo G et al. A novel homozygous nonsense deletion/insertion mutation in the keratin 14 gene causes recessive epidermolysis bullosa simplex type Koebner. Exp Dermatol 2003; 28:77-9.
18 Yasukawa K, Sawamura D, McMillan JR et al. Dominant and recessive compound heterozygous mutations in epidermolysis bullosa simplex demonstrate the role of the stutter region in keratin intermediate filament assembly. J Biol Chem 2002; 277:23670-4.

19 Patterson DF. Companion animal medicine in the age of medical genetics. J Vet Intern Med 2000; 14:1-9.

20 McKusick VA. Mendelian Inheritance in Man: a Catalog of Human Genes and Genetic Disorders, 11th edn. Baltimore, MD: The Johns Hopkins University Press, 1994.

21 Reichelt J, Bussow H, Grund C et al. Formation of a normal epidermis supported by increased stability of keratins 5 and 14 in keratin 10 null mice. Mol Bio Cell 2001; 12:1557-68.

22 Reichelt J, Magin TM. Hyperproliferation, induction of $\mathrm{c}-\mathrm{Myc}$ and 14-3-3 $\sigma$, but no cell fragility in keratin-10-null mice. J Cell Sci 2002; 115:2639-50.

23 Arin MJ, Longley MA, Wang X-J et al. Focal activation of a mutant allele defines the role of stem cells in mosaic skin disorders. J Cell Biol 2001; 152:645-9. 